\title{
ECLETICA
}

www.scielo.br/eq

www.ecletica.iq.unesp.br

Volume 33, número 1, 2008

\section{Estudo do comportamento eletroquímico da enzima peroxidase na presença de peróxido de hidrogênio e ácido 5- aminossalicílico}

\author{
C. V. Uliana, C. S. Riccardi, H. Yamanaka* \\ Universidade Estadual Paulista, Instituto de Química, Campus de Araraquara, CP 355, 14800-900, Araraquara, SP. \\ *hidekoy@iq.unesp.br
}

\begin{abstract}
Resumo: O comportamento eletroquímico da enzima peroxidase (HRP) foi estudado utilizando o peróxido de hidrogênio como substrato enzimático e o ácido 5-aminossalicílico (5-ASA) como mediador de elétrons sobre eletrodo de grafite. Diversos parâmetros foram otimizados, tais como, o potencial aplicado à técnica amperométrica fixado em $-0,125 \mathrm{~V}$, a solução tampão fosfato-citrato $0,1 \mathrm{~mol} \mathrm{~L}$ $1 \mathrm{pH}$ 5,0 como eletrólito suporte e a proporção entre o 5-ASA e $\mathrm{H}_{2} \mathrm{O}_{2}$ em 1:7, entre outros. Foi observada a catálise da reação de oxidação do peróxido de hidrogênio na presença da enzima HRP e do mediador 5-ASA. O produto dessa oxidação foi reduzido na superfície do eletrodo, evidenciando um significativo aumento na intensidade da corrente catódica.
\end{abstract}

Palavras-chave: peroxidase; peróxido de hidrogênio; ácido 5-aminossalicílico; eletrodo de grafite.

\section{Introdução}

A enzima peroxidase (HRP, EC: 1.11.1.7, peróxido de hidrogênio oxidorredutase), proveniente de raiz de rábano silvestre (horseradish root), é freqüentemente empregada em química analítica por manter uma resposta estável por longos períodos de tempo a temperatura ambiente e em um amplo intervalo de $\mathrm{pH}$, além de ter custo financeiro relativamente baixo e pode ser encontrada comercialmente em diversos graus de pureza [1].

A HRP é uma glicoproteína consistida de 308 resíduos de aminoácidos, dois $\mathrm{Ca}^{2+}$ e como grupo prostético (sítio ativo da enzima) uma ferroprotoporfirina IX, sendo denominado grupo heme, que está ligado não covalentemente à cadeia polipeptídica. $O$ íon férrico central da referida protoporfirina está coordenado a um resíduo de histidina. As propriedades catalíticas, estruturais e eletroquímicas das peroxidases foram revisadas por Ruzgas e col. [2].

Esta enzima catalisa a redução de peróxidos, por meio de um mecanismo complexo de reações [3]:

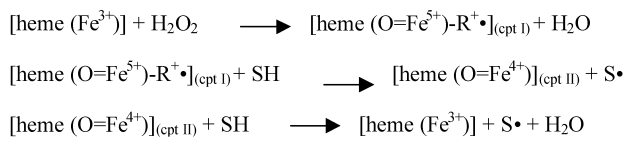

A equação 1 envolve a oxidação do grupo prostético heme da peroxidase pelo peróxido de hidrogênio (ou hidroperóxidos orgânicos) por dois elétrons, e a formação de um composto intermediário, no estado ( $\mathrm{Fe}^{5+}$, Composto I), consistindo do ferro oxiferril $\left(\mathrm{O}=\mathrm{Fe}^{5+}\right)$ e do cátion radical $\pi$ porfirínico. $\mathrm{Na}$ equação 2 , o composto 
intermediário $\left(\mathrm{Fe}^{5+}\right)$ formado sofre uma reação de redução pela transferência de um elétron do doador SH, formando composto intermediário, no estado de oxidação ( $\mathrm{Fe}^{4+}$, Composto II). Um elétron adicional pode ser transferido, proveniente de uma segunda molécula $\mathrm{AH}$ (Equação 3 ), de forma que a enzima retorne a forma nativa $\left(\mathrm{Fe}^{3+}\right)$. Nas etapas 2 e 3, a espécie doadora de elétrons (SH) é oxidada.

Quando o eletrodo substitui o substrato doador de elétrons em um ciclo comum da reação do peróxido, o processo é denominado transferência eletrônica direta. Enzimas imobilizadas no eletrodo podem ser oxidadas pelo peróxido (equação 1) e então reduzidas por elétrons provenientes do eletrodo (equação 4).

$$
\left[\text { heme }\left(\mathrm{O}=\mathrm{Fe}^{5+}\right)-\mathrm{R}+\bullet\right]_{(\text {ppt I })}+2 \mathrm{e}^{-}+\mathrm{H}^{+} \longrightarrow\left[\text { heme }\left(\mathrm{Fe}^{3+}\right)\right]+\mathrm{H}_{2} \mathrm{O}
$$

A transferência eletrônica direta entre a HRP adsorvida em eletrodos tem sido demonstrada em publicações desde 1978 [4]. Vários materiais eletródicos têm sido empregados para estabilizar a transferência eletrônica direta da HRP, entre eles, carbono vítreo [5], grafite [6], ouro modificado [7] e platina [8]. No entanto, em geral, esse processo é lento. Assim, um mediador de transferência de elétrons tem sido usado para melhorar a velocidade de transferência eletrônica, sendo mais eficiente na redução bioeletrocatalítica de peróxidos em eletrodos modificados com HRP.

Quando um doador de elétrons (S) está presente num sistema peroxidase-eletrodo, os processos diretos e mediados podem ocorrer simultaneamente com a redução do doador $\mathrm{S} \bullet$ oxidado pelo eletrodo (equação 5).

$$
\mathrm{S} \bullet+\mathrm{e}^{-}+\mathrm{H}^{+} \longrightarrow \mathrm{SH}
$$

Os mediadores são pequenas moléculas passíveis de redução/oxidação com alta velocidade de transferência eletrônica que distribuem os elétrons da superfície do eletrodo para os compostos I e II. A molécula do mediador escolhido deve reagir rapidamente com a peroxidase oxidada. Uma variedade de moléculas doadoras de elétrons pode reagir rapidamente com os compostos I e II, sendo que, para a enz- ima HRP pode-se utilizar como mediador de elétrons: hidroquinona [9, 10], ácido ascórbico [11], ferroceno [12,13], iodeto [14, 15, 16], entre outros. O ácido 5-aminossalicílico (5ASA) também tem sido usado como mediador para a enzima HRP em imunoensaios com detecção ótica [17], potenciométrica [18] e amperométrica $[19,20,21]$, sendo que nos trabalhos utilizando a técnica amperométrica, a HRP foi imobilizada na superfície dos diferentes eletrodos.

O presente trabalho apresenta um estudo sobre o comportamento eletroquímico da enzima HRP sobre eletrodo de grafite utilizando o peróxido de hidrogênio como substrato enzimático e o 5-ASA como mediador da transferência eletrônica.

\section{Materiais e métodos}

\section{Equipamentos e Reagentes}

As medidas eletroquímicas foram realizadas empregando-se um PotenciostatoGalvanostato PAR EG\&G modelo 263. Uma célula eletroquímica de compartimento único de $5 \mathrm{~mL}$ foi utilizada para as medidas, juntamente com um sistema de três eletrodos: eletrodo de trabalho (eletrodos de grafite, perfazendo uma área de $\left.0,00318 \mathrm{~cm}^{2}\right)$, eletrodo de referência $(\mathrm{Ag} / \mathrm{AgCl})$ e o eletrodo auxiliar (Pt, área de $\left.1 \mathrm{~cm}^{2}\right)$.

A enzima peroxidase (987 unidades HRP/mg proteína) foi fornecida pela Sigma; fosfato de sódio monobásico, ácido cítrico e fosfato de sódio dibásico pela Mallinckrodt, peróxido de hidrogênio estabilizado fornecido pela Merck e ácido 5-aminossalicílico (5-ASA) pela Acros. Empregou-se o sistema Milli-Q Ultra Pure Water System da marca Millipore, para a obtenção de água ultrapura.

A solução tampão fosfato de sódio $0,1 \mathrm{~mol}$ $\mathrm{L}^{-1} \mathrm{pH}$ 7,0 foi preparada a partir dos sais $\mathrm{Na}_{2} \mathrm{HPO}_{4} / \mathrm{NaH}_{2} \mathrm{PO}_{4}$. As soluções tampão fosfatocitrato $0,1 \mathrm{~mol} \mathrm{~L}^{-1} \mathrm{pH} \mathrm{5,0}$ foram adequadamente preparadas a partir dos respectivos sais segundo Gomori [22]. Para a preparação da solução tampão Britton-Robinson utilizou-se duas soluções, sendo a solução 1 composta de uma mistura de 4,0 mol L-1 dos ácidos ortofosfórico, acético e 
bórico e a solução 2 de hidróxido de sódio 1,0 mol L-1 empregada para ajuste do $\mathrm{pH}^{23}$. A enzima HRP foi dissolvida em água deionizada $\left(C_{\mathrm{f}}=2,0\right.$ $\mathrm{mg} \mathrm{mL} \mathrm{mL}^{-1}$ de proteína), sendo a solução armazenada $\mathrm{a}-4^{\circ} \mathrm{C}$ como solução estoque. $\mathrm{O}$ monitoramento da atividade da HRP foi realizado empregando-se soluções de $\mathrm{H}_{2} \mathrm{O}_{2}$ e 5-ASA, preparadas imediatamente antes do uso, em água deionizada, à temperatura de $(25 \pm 1)^{\circ} \mathrm{C}$.

\section{Resultados e discussão}

Comportamento eletroanalítico do 5-ASA na presença de $\mathrm{H}_{2} \mathrm{O}_{2}$ e da HRP.

Um estudo por voltametria cíclica foi realizado empregando-se o intervalo de potencial de $+0,250 \mathrm{~V}$ a $-0,300 \mathrm{~V}$ e $v=0,050 \mathrm{~V} \mathrm{~s}^{-1}$. O monitoramento da reação catalisada ou não pela HRP foi conduzido em solução tampão fosfato-citrato pH 5,0 na presença de $\mathrm{H}_{2} \mathrm{O}_{2} 1,0 \times 10^{-3} \mathrm{~mol} \mathrm{~L}^{-1} \mathrm{e}$ 5-ASA $1,0 \times 10^{-3} \mathrm{~mol} \mathrm{~L}^{-1}$.

$\mathrm{O}$ voltamograma cíclico referente à reação do 5-ASA apresentou dois picos catódicos $\left(\mathrm{E}_{\mathrm{pc} 1}=\right.$ $\left.0,067 \mathrm{~V} / \mathrm{E}_{\mathrm{pc} 2}=-0,107 \mathrm{~V}\right)$ e dois picos anódicos $\left(\mathrm{E}_{\mathrm{pa} 1}=0,125 \mathrm{~V} / \mathrm{E}_{\mathrm{pa} 2}=-0,080 \mathrm{~V}\right)$, como pode ser observado na Figura 1B. A oxidação do 5-ASA é um processo com transferência de dois elétrons para formação das espécies eletroquimicamente ativas 5-ASA quinoneimina (5-ASA-QI). A

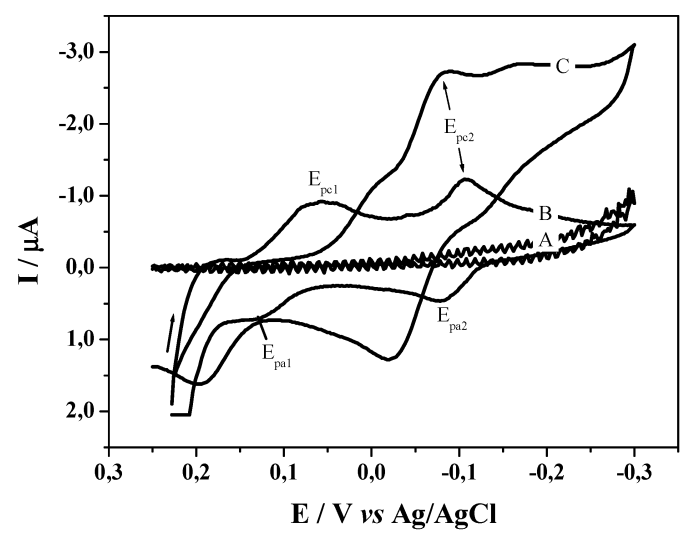

Figura 1. Voltamogramas cíclicos obtidos em solução tampão fosfato-citrato $0,1 \mathrm{~mol} \mathrm{~L}^{-1} \mathrm{pH} 5,0$ contendo $\mathrm{H}_{2} \mathrm{O}_{2} 1,0 \times 10^{-3} \mathrm{~mol} \mathrm{~L}^{-1}$ e HRP $1,0 \mathrm{U} \mathrm{mL}^{-1}$ (A); $\mathrm{H}_{2} \mathrm{O}_{2} /$ 5-ASA 1,0 x 10-3 $\mathrm{mol} \mathrm{L}^{-1}$ na ausência (B) e na presença (C) de HRP 1,0 U mL-1 em solução; $v=0,050 \mathrm{~V} \mathrm{~s}^{-1}$. quinoneimina pode ser hidrolizada a benzoquinona que forma o par redox quinona/hidroquinona [24].

Na presença da HRP, o 5-ASA oxidado pela catálise enzimática $\left(5-\mathrm{ASA}_{\mathrm{ox}}\right)$, é detectado na superfície do eletrodo por meio de técnicas eletroanalíticas. Observou-se um aumento significativo da intensidade de corrente do pico catódico para o sistema $\mathrm{H}_{2} \mathrm{O}_{2}$ / 5-ASA; valor de $\mathrm{I}_{\mathrm{pc} 2}$ de -1,22 $\mu \mathrm{A}$ na ausência da HRP (Figura 1B) para $-2,72 \mu \mathrm{A}$ na presença da enzima (Figura $1 \mathrm{C}$ ). Quando a medida foi realizada na solução contendo $\mathrm{H}_{2} \mathrm{O}_{2} \mathrm{e}$ HRP na ausência de 5-ASA, o valor da intensidade de corrente foi baixo (Figura 1A).

Efeito do valor de potencial aplicado na técnica amperométrica.

Transientes de $+0,100 \mathrm{~V}$ a $-0,250 \mathrm{~V} v s$. $\mathrm{Ag} / \mathrm{AgCl}$ foram efetuados para avaliar o efeito do potencial aplicado no comportamento do sistema enzimático. A Figura 2 mostra que, na presença da enzima HRP, houve um aumento do valor de intensidade de corrente catódica em função do potencial aplicado de $+0,100$ a $-0,125 \mathrm{~V}$, mantendo-se constante a partir deste potencial. Na ausência da HRP, a intensidade de corrente nesse intervalo de potencial é praticamente constante e próximo de zero. Portanto, foi definido o potencial de $-0,125 \mathrm{~V}$ para o monitoramento da atividade enzimática.

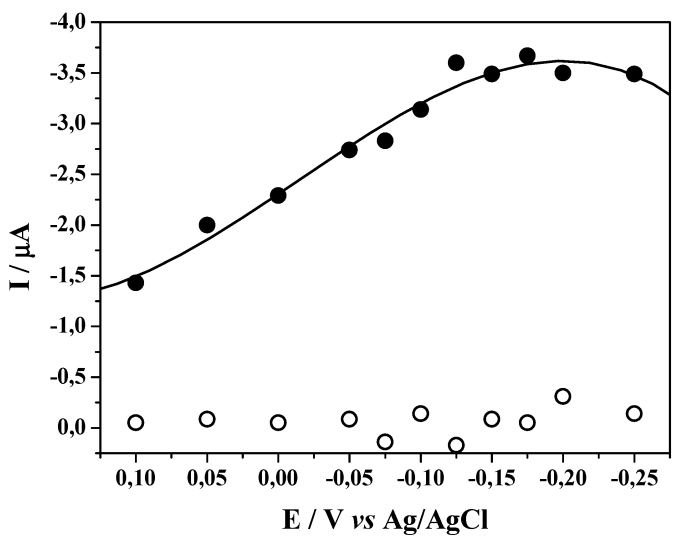

Figura 2. Efeito do valor de potencial aplicado no monitoramento amperométrico do sistema 5-ASA / $\mathrm{H}_{2} \mathrm{O}_{2}$ (ambos na concentração $1,0 \times 10^{-3} \mathrm{~mol} \mathrm{~L}^{-1}$ ) em solução tampão Britton-Robinson $0,1 \mathrm{~mol} \mathrm{~L}^{-1} \mathrm{pH} 6,9$, na ausência (O) e na presença (n) de $1,0 \mathrm{U} \mathrm{mL}^{-1}$ da enzima HRP. 
Influência do $\mathrm{pH}$.

A enzima HRP em solução apresentou atividade em uma ampla faixa de $\mathrm{pH}$ entre 4,0 e 8,0 (Figura 3), sendo que o valor de intensidade

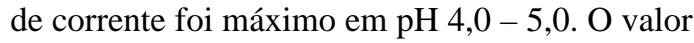
de $\mathrm{pH}$ igual a 5,0 foi escolhido para o monitoramento amperométrico da atividade enzimática nos experimentos subseqüentes.

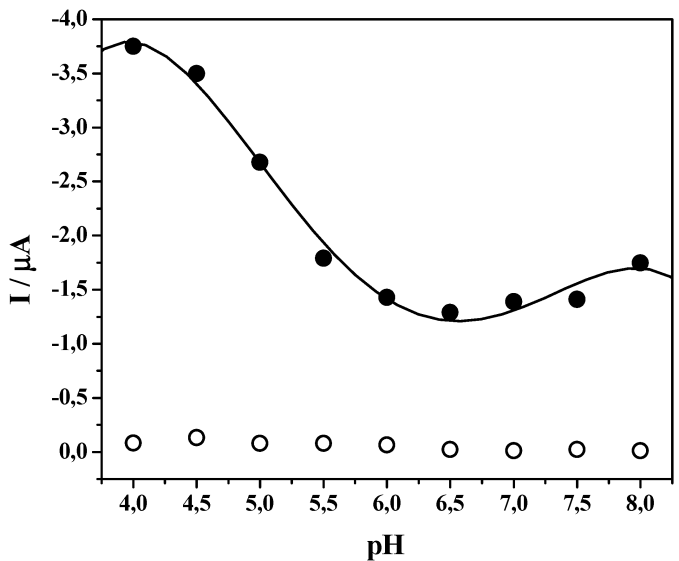

Figura 3. Efeito do valor de $\mathrm{pH}$ no monitoramento amperométrico da reação enzimática em solução tampão Britton-Robinson $0,1 \mathrm{~mol} \mathrm{~L}^{-1}$ contendo 5ASA/ $\mathrm{H}_{2} \mathrm{O}_{2}$ (ambos na concentração $1,0 \times 10^{-3} \mathrm{~mol} \mathrm{~L}^{-1}$ ), na ausência $(O)$ e na presença $(\bullet)$ da HRP $1,0 \mathrm{U} \mathrm{mL}^{-1}$. $\mathrm{E}_{\mathrm{apl}}=-0,125 \mathrm{~V} v s . \mathrm{Ag} / \mathrm{AgCl}$.

\section{Efeito do eletrólito de suporte}

Avaliou-se a influência do eletrólito de suporte na resposta enzimática empregando-se as seguintes soluções tampão $0,1 \mathrm{~mol} \mathrm{~L}^{-1} \mathrm{pH} \mathrm{5,0:}$ citrato de sódio, Britton-Robinson, acetato de sódio, fosfato-citrato e fosfato de sódio. A atividade da enzima foi monitorada a $-0,125 \mathrm{~V}$ empregando-se o sistema 5-ASA / $\mathrm{H}_{2} \mathrm{O}_{2}$ (ambos na concentração $\left.1,0 \times 10^{-3} \mathrm{~mol} \mathrm{~L}^{-1}\right)$.

A Figura 4 indica que, para o sistema contendo 5-ASA / $\mathrm{H}_{2} \mathrm{O}_{2}$ na ausência da HRP em solução tampão fosfato-citrato, o valor de intensidade de corrente é menor quando comparado às demais soluções, enquanto que, na presença da HRP há significativo aumento de corrente. Portanto, a solução tampão fosfato-citrato de sódio foi escolhida para o monitoramento da atividade da enzima HRP.

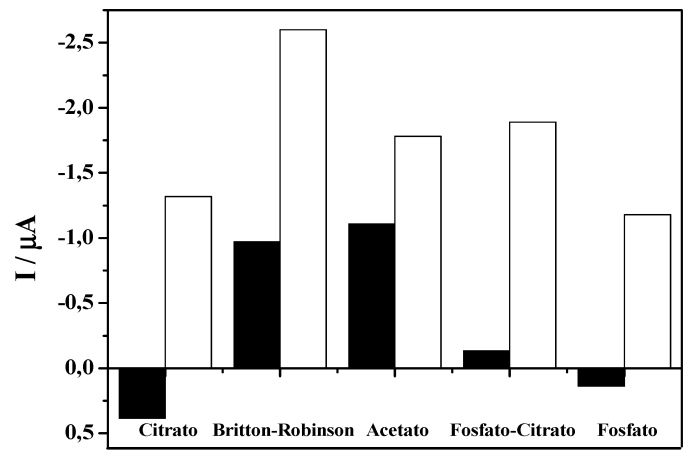

Figura 4. Gráfico relativo à intensidade de corrente obtida por amperometria a $-0,125 \mathrm{~V}$ vs. $\mathrm{Ag} / \mathrm{AgCl}$, para o monitoramento do sistema $\mathrm{H}_{2} \mathrm{O}_{2} / 5$-ASA na ausência (preto) e na presença (cinza) da enzima HRP $1,0 \mathrm{U} \mathrm{mL}^{-1}$, em diferentes eletrólitos de suporte.

Efeito da proporção entre os substratos 5-ASA/ $\mathrm{H}_{2} \mathrm{O}_{2}$

Inicialmente, para estudar o efeito da proporção $\mathrm{H}_{2} \mathrm{O}_{2} / 5$-ASA, avaliou-se a resposta enzimática fixando a concentração de $\mathrm{H}_{2} \mathrm{O}_{2}$ em $0,5 \mathrm{x}$ $10^{-3} \mathrm{~mol} \mathrm{~L}^{-1}$ frente a um aumento da concentração de 5-ASA (0,250;0,750;1,00; 1,50 e 2,00 x 10-3 mol L-1). A catálise enzimática foi observada para os substratos $\mathrm{H}_{2} \mathrm{O}_{2} / 5$-ASA nas proporções 1:0,5; $1: 1,0$ e $1: 1,5$. Porém, o valor de intensidade de corrente catódica diminui com o aumento da concentração de 5-ASA na presença da enzima HRP e, conforme descrito na literatura ${ }^{20}$, concentrações elevadas de 5-ASA podem induzir a um bloqueio parcial da superfície do eletrodo pela forma oxidada do substrato enzimático. Deste modo, avaliouse a resposta enzimática fixando a concentração do 5-ASA em 2,50 x $10^{-4} \mathrm{~mol} \mathrm{~L}^{-1}$ frente a um aumento da concentração de $\mathrm{H}_{2} \mathrm{O}_{2}$. A resposta enzimática máxima ocorreu quando a proporção para os substratos 5-ASA $/ \mathrm{H}_{2} \mathrm{O}_{2}$ foi de 1:7. Para concentrações elevadas de $\mathrm{H}_{2} \mathrm{O}_{2}$ houve um aumento do valor de intensidade de corrente catódica para o sistema na ausência da enzima HRP, não sendo viável o monitoramento da atividade da enzima acima da proporção de 1:7 entre 5-ASA/ $\mathrm{H}_{2} \mathrm{O}_{2}$.

Estudo do tempo de incubação dos eletrodos na solução contendo 5-ASA/ $\mathrm{H}_{2} \mathrm{O}_{2} / \mathrm{HRP}$ antes das medidas amperométricas.

Seqüencialmente, avaliou-se a variação da intensidade de corrente devido a um aumento 
do tempo de incubação dos eletrodos na solução contendo 5-ASA $/ \mathrm{H}_{2} \mathrm{O}_{2}$ na ausência e na presença da HRP (Figura 5). Na ausência da enzima, observou-se um ligeiro aumento do valor de intensidade de corrente que se estabiliza a partir de 10 minutos até pelo menos 30 minutos de incubação. Na presença de 1,0 $\mathrm{U} \mathrm{mL}^{-1}$ de $\mathrm{HRP}$, ocorreu um aumento significativo do valor de intensidade de corrente a partir de 2 minutos de incubação da reação, tornando-se constante a partir desse tempo. Este tempo de incubação é relativamente curto, quando comparado ao sistema que utiliza o iodeto como mediador, por exemplo, cujo tempo de incubação é de 10 minutos antes das medidas [14,15]. Adotou-se para todos os experimentos, um tempo de incubação do sistema 5-ASA $/ \mathrm{H}_{2} \mathrm{O}_{2}$ de 2 minutos antes do início das medidas eletroanalíticas.

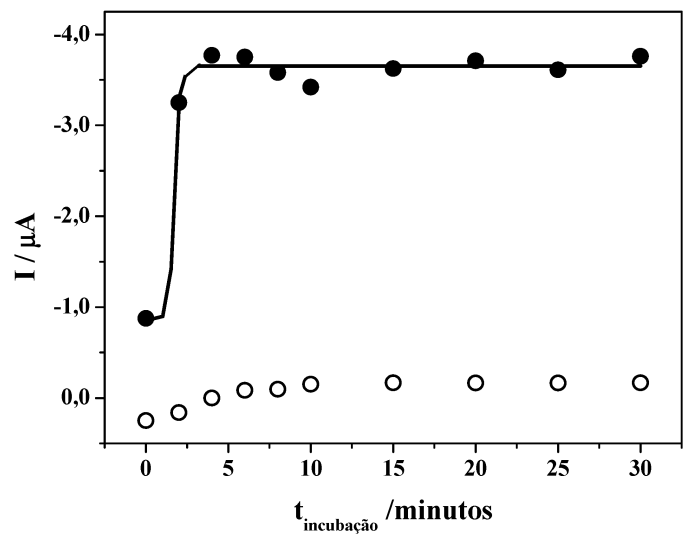

Figura 5: Valores de intensidade de corrente em função do tempo de incubação do sistema 5-ASA/ $\mathrm{H}_{2} \mathrm{O}_{2}$ 1:7 em solução tampão fosfato-citrato de sódio $0,1 \mathrm{~mol} \mathrm{~L}^{-1} \mathrm{pH}$ 5,0 na ausência $(\bigcirc)$ e na presença $(\bullet)$ da enzima HRP $1,0 \mathrm{U} \mathrm{mL} \mathrm{mL}^{-1}$ para concentrações dos substratos enzimático de $0,50 \times 10^{-3} \mathrm{~mol} \mathrm{~L}^{-1} 5$-ASA e $3,5 \times 10^{-3} \mathrm{~mol} \mathrm{~L}^{-1} \mathrm{de}$ $\mathrm{H}_{2} \mathrm{O}_{2}$. O sistema operou a $-0,125 \mathrm{~V}$ vs. $\mathrm{Ag} / \mathrm{AgCl}$.

\section{Curva analítica da enzima HRP}

A curva analítica da $\operatorname{HRP}(0,0$ a $43 \mu \mathrm{g} \mathrm{mL}-1)$, em diferentes concentrações dos substratos 5ASA/ $\mathrm{H}_{2} \mathrm{O}_{2}$ proporção 1:7, dentro dos pontos da linearidade (concentração 5-ASA/ $\mathrm{H}_{2} \mathrm{O}_{2}$ : a) 0,125 e 0,875 , b) 0,250 e 1,75 , c) 0,500 e 3,50 ; d) 1,00 e 7,00 x $10^{-3} \mathrm{~mol} \mathrm{~L}^{-1}$, respectivamente) do sistema é mostrada na Figura 6. Os valores de intensidade de corrente catódica aumentaram proporcionalmente à concentração de HRP adicionada ao sistema para todas as concentrações estudadas. Pode-se observar que a resposta enzimática apresentou o maior intervalo linear, de 0,10 a $13 \mu \mathrm{g} \mathrm{mL}-1$, para concentrações de $0,50 \times 10^{-3} \mathrm{~mol} \mathrm{~L}^{-1}$ de 5-ASA e 3,50 x $10^{-3} \mathrm{~mol} \mathrm{~L}^{-1}$ de $\mathrm{H}_{2} \mathrm{O}_{2}$.

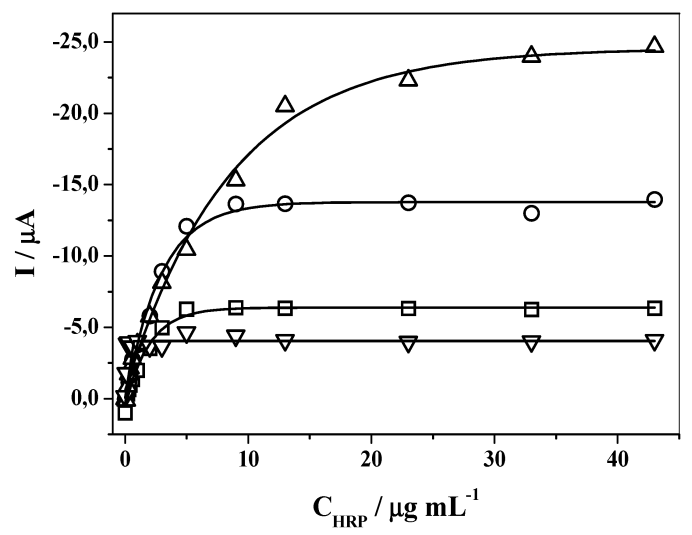

Figura 6. Curva analítica para variação da concentração de HRP em diferentes concentrações dos substratos 5-ASA/ $\mathrm{H}_{2} \mathrm{O}_{2}:(\square) 0,125$ e 0,875 , (O) 0,250 e $1,75,(\Delta) 0,500$ e 3,$50 ;(\nabla) 1,00$ e $7,00 \times 10^{-3} \mathrm{~mol} \mathrm{~L}^{-1}$, respectivamente.

A otimização das condições experimentais para o monitoramento da atividade catalisadora da enzima HRP possibilita o emprego da metodologia no desenvolvimento de eletrodos modificados com a enzima para aplicação em biossensores.

\section{Conclusões}

A enzima HRP catalisa a reação de oxidação do mediador de elétrons ácido 5-aminossalicílico sobre eletrodo de grafite, evidenciado pelo significativo aumento da intensidade de

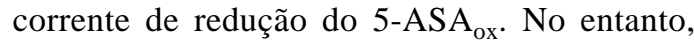
em altas concentrações do substrato $\mathrm{H}_{2} \mathrm{O}_{2}$ e do 5-ASA, ocorre a inibição do sítio catalítico da enzima. $\mathrm{O}$ potencial de $-0,125 \mathrm{~V}$ aplicado na técnica amperométrica, inferior aos encontrados na literatura para outros mediadores que 
variam de $-0,150$ a $-0,400 \mathrm{~V}$, apresenta a vantagem de diminuir a interferência de outras espécies que podem ser reduzidas na superfície do eletrodo. Desta maneira, foi possível determinar as condições experimentais ótimas para o monitoramento da atividade catalisadora da enzima HRP.

\section{Agradecimentos}

Os autores agradecem à FAPESP pelo suporte financeiro.

Recebido em 25 de fevereiro de 2008

Aceito em 27 de março de 2008

C. V. Uliana, C. S. Riccardi, H. Yamanaka. Investigation on electrochemical behavior of peroxidase enzyme in the presence of hydrogen peroxide and 5-aminosalicylic acid.

Abstract: The electrochemical behavior of the enzyme peroxidase (HRP) was investigated using the hydrogen peroxide as enzymatic substrate and the 5-aminosalicylic acid (5-ASA) as mediator of electrons on graphite electrodes. Several parameters were optimized, namely, the applied potential to the amperometric technique fixed in $-0.125 \mathrm{~V}$, the $0.1 \mathrm{~mol} \mathrm{~L}^{-1}$ phosphate-citrate buffer at $\mathrm{pH} 5.0$ as supporting electrolyte and the proportion between the 5-ASA and $\mathrm{H}_{2} \mathrm{O}_{2}$ in 1:7, among others. It was observed the catalysis of the oxidation reaction of the $\mathrm{H}_{2} \mathrm{O}_{2}$ in the presence of the enzyme HRP and 5ASA. The oxidation product was reduced in the electrode surface, evidencing a significant increase in the intensity of the cathodic current.

Keywords: peroxidase; hydrogen peroxide; 5-aminosalicilic acid; graphite electrode.

\section{Referências}

[1] H. B. DUNFORD, Horseradish Peroxidase: Structures and kinetics Properties. In: Everse J.; Everse, K. E.; Grishan, M. B. (Eds.) Peroxidases in Chemistry and Biology, CRC Press, Boca Raton, FL (1991) 2.

[2] T. Ruzgas, E. Csoregi, J. Emneus, L. Gorton, G. M. Varga, Anal. Chim. Acta, 330 (2-3) (1996) 123.

[3] R. S. Freire, C. A. Pessoa, L. D. Mello, L. T. Kubota, J. Braz. Chem. Soc. 14(2) (2003) 230.

[4] A. I. Yaropolov, M. R. Tarasevich, S. D. Varfolomeev, Bioelectochem. Bioenerg. 5 (1) (1978) 18.

[5] Q. Lu, X. Chen, Y. Wu, S. Hu, Biophys. Chem. 117 (1) (2005) 55.

[6] Y. Zhang, P. He, N. Hu, Electrochim. Acta 49 (12) (2004) 1981.

[7] J. Hong, A. A. Moosavi-Movahedi, H. Ghourchian, A. M. Rad, S. Rezaei-Zarchi, Electrochim. Acta 52 (21) (2007) 6261.

[8] Y. Liu, R. Yuan, Y. Chai, D. Tang, J. Dai, X. Zhong, Sens. Actuators B 115 (1) (2006) 109.

[9] G. A. Messina, A. A. J. Torriero, I. E. De Vito, J. Raba, Talanta 64 (4) (2004) 1009.

[10] S. Sánchez, M. Pumera, E. Fábregas, Biosens. Bioelectron. 23 (3) (2007) 332.

[11] N.F. Starodub, B.B. Dzantiev, V.M. Starodub, A.V. Zherdev, Anal. Chim. Acta 424 (1) (2000) 37.

[12] Y. Okawa, M. Nagano, S. Hirota, H. Kobayashi, T. Ohno, M. Watanabe, Biosens. Bioelectron. 14 (2) (1999) 229.
[13] S. Liawruangrath, W. Oungpipat, S. Watanesk, B. Liawruangrath, C. Dongduend, P. Purachat, Anal. Chim. Acta 448 (1-2) (2001) 37.

[14] A. A. P. Ferreira, W. Colli, P. I. Costa, H. Yamanaka, Biosens. Bioelectron. 21 (1) (2005) 175.

[15] A. A. P. Ferreira, W. Colli, M. J. M. Alves, D. R. Oliveira, P. I. Costa, A. G. Guell, F. Sanz, A.V. Benedetti, H. Yamanaka, Electrochim. Acta 51 (24) (2006) 5046.

[16] C. S. Riccardi, K. Dahmouche, C. V. Santilli, P. I. Costa, H. Yamanaka, Talanta 70 (3) (2006) 637.

[17] N. V. Girina, E. I. Karaseva, D. I. Metelitsa, Biochem. Moscol, 59 (12) (1994) 1397.

[18] B. B. Dzantiev, A. V. Zherdev, M. F. Yulaev, R. A. Sitdikov, N. M. Dmitrieva, I. Y. Moreva, Biosens. Bioelectron. 11 (1-2) (1996) 179.

[19] I. Abdel-Hamid, D. Ivnitski, P. Atanasov, E. Wilkins, Electroanal. 10 (11) (1998) 758.

[20] I. Eshkenazi, E. Maltz, B. Zion, J.Rishpon, J. Dairy Scien. 83 (9) (2000) 1939.

[21] R. Solna, P. Skladal, S. A. Eremin, Int. J. Environ. Anal. Chem. 83 (7-8) (2003) 609.

[22] G.Gomorri, General Preparative Procedures, In: Methods in Enzymology, S.P. Colowick and N. O. Caplan (Eds.) Academic Press, NY (1965) 142.

[23] H. T. S. Britton, R.A. J. Robinson, Chem. Soc. 458 (1931) 287.

[24] M. R Tarasevich, A. I. Yaropolov, V. A. Bogdanovskaya, S. D. Varfolomeev, Bioelectrochem. Bioenerg. 6 (3) (1979) 393. 